\title{
Calibration of Undrained Shear Strength Partial Factor Using Probability Theory
}

\author{
Filip Dodigović*, Krešo Ivandić, Božo Soldo, Kristijan Grabar
}

\begin{abstract}
Design in accordance with Eurocode 7 applies the partial factors of geotechnical parameters to take into account uncertainties of various sources. The prescribed values of partial factors are unique, even though the degree of uncertainty can vary significantly. This can lead to an unequal reliability level of structures designed using the same procedures and methods. This paper analyses the influence of undrained shear strength variability on the reliability index $(\beta)$, using reliability theory and statistical methods. Analyses were performed in the case of a shallow footing designed in accordance with Eurocode 7, design approach 3 . It is shown that reliability indexes of a shallow footing could be lower than the target values prescribed in Eurocode. To meet these values, additional elaboration and calibration of undrained shear strength partial factor $\left(\gamma_{\mathrm{cu}}\right)$ was proposed.
\end{abstract}

Keywords: Eurocode 7; geotechnical design; partial factor; reliability analysis; uncertainty; undrained shear strength

\section{INTRODUCTION}

The measure of safety of geotechnical structures designed in accordance with Eurocode is regulated by the application of partial factors (PF) to actions, materials, and resistances. In this way, instead of one uncertainty applied to the whole model, they are considered separately for each component of that model [1]. The influence of PF values on structural reliability has been investigated by several authors [2-6]. These studies can generally be divided into two groups: direct studies, which directly calculate the reliability of structures using the prescribed values of $\mathrm{PF}$, and indirect studies, which calibrate PF values to achieve the target value of the reliability index $(\beta)$ [7]. The reliability index is defined as the negative value of the inverse Gaussian distribution of probability failure. Calibration of PF values can be performed using reliability methods [8]. However, almost all values used in modern codes have been calibrated against previous successful experience, with very little use of reliability methods [9].

One of Eurocode's objectives is to provide a similar level of structural reliability, regardless of the relationship between actions, material strengths, and resistances. Tab. 1 shows the three reliability classes associated with the recommended minimum reliability index $(\beta)$ values for two reference periods [8].

Table 1 Recommended minimum values for reliability index for ULS

\begin{tabular}{|c|c|c|}
\hline \multirow{2}{*}{ Reliability Class } & \multicolumn{2}{|c|}{ Minimum values for $\beta$} \\
\cline { 2 - 3 } & 1 year reference period & 50 years reference period \\
\hline RC3 & 5.2 & 4.3 \\
\hline RC2 & 4.7 & 3.8 \\
\hline RC1 & 4.2 & 3.3 \\
\hline
\end{tabular}

A common reliability class considered in everyday practice is the $\mathrm{RC} 2$, which includes residential, commercial, and public buildings with medium failure consequences (e.g. office buildings). Reliability indexes shown in Tab. 1 are recommended minimum values for structures designed in accordance with the Eurocodes.

It can be noted that the reliability indexes for a lower reference period are higher, which may seem illogical at first glance. In geotechnical engineering, this can be interpreted as the influence of time on soil degradation due to softening. This problem is addressed by [10]. In the example of a retaining wall, they showed that due to soil degradation, the reliability index would decrease from 4.7 to 3.8 over a period of 30-40 years. Analyses were performed using partial factors, which were calibrated to a reference period of 50 years.

Meyerhof [11] claims that the probability of failure of retaining structures and foundations, designed with the typical values of the overall factor of safety, is $10^{-3}$ to $10^{-4}$ $(3.1<\beta<3.7)$, which he finds satisfactory.

Compared to Eurocode 7, which prescribes a unique partial factors value, the Canadian Standards Organization [12] distinguishes their values with respect to the "degree of understanding". The "degree of understanding" adjusts resistance factors as a function of site and model understanding. Concerning the above, three classes are defined: low, typical, and high "degree of understanding". A similar categorization of site variability in piles bearing capacity analysis, based on the values of the coefficient of variation $(\mathrm{COV})$ of geotechnical parameters, is proposed by Paikowsky et al. [13]: low $(C O V<25 \%)$, medium $(25 \% \leq$ $C O V<40 \%)$ and high $(C O V \geq 40 \%)$ site variability. The coefficient of variation is defined as the ratio between the standard deviation and the mean value of a random variable.

Based on several calibration studies, Phoon [14] proposes three categories of parameter variability (low, medium, and high) to achieve reasonable, uniform reliability levels of geotechnical structures (Tab. 2).

Table 2 Ranges of soil property variability for reliability calibration [14]

\begin{tabular}{|c|c|c|}
\hline Geotechnical parameter & Property variability & $C O V(\%)$ \\
\hline \multirow{3}{*}{ Undrained shear strength } & Low $^{\mathrm{a}}$ & $10-30$ \\
\cline { 2 - 3 } & Medium $^{\mathrm{b}}$ & $30-50$ \\
\cline { 2 - 3 } & High $^{\mathrm{c}}$ & $50-70$ \\
\hline \multirow{3}{*}{ Effective stress friction angle } & Low $^{\mathrm{a}}$ & $5-10$ \\
\cline { 2 - 3 } & Medium $^{\mathrm{b}}$ & $10-15$ \\
\cline { 2 - 3 } & High $^{\mathrm{c}}$ & $15-20$ \\
\hline \multirow{3}{*}{ Horizontal stress coefficient } & Low $^{\mathrm{a}}$ & $30-50$ \\
\cline { 2 - 3 } & Medium $^{\mathrm{b}}$ & $50-70$ \\
\cline { 2 - 3 } & High $^{\mathrm{c}}$ & $70-90$ \\
\hline
\end{tabular}

${ }^{a}$ typical of good quality direct lab or field measurements; ${ }^{b}$ typical of indirect correlations with good field data, except for the standard penetration test (SPT); ${ }^{c}$ typical of indirect correlations with SPT field data and with strictly empirical correlations. 


\section{STATEMENT OF THE PROBLEM}

Regardless of the uncertainty degree, Eurocode 7 prescribes a unique value of a partial factor for a geotechnical parameter. The consequence is an unequal level of structural reliability, which does not correspond to the initial Eurocode's intention to ensure an equal reliability level.

In addition to the above, results presented by several authors $([3,6])$ indicate that the reliability index $(\beta)$ of shallow foundations designed in accordance with EC7 could be lower than values prescribed in Tab. 1. Murakami et al. [6] investigated the relationship between the PF and $\beta$ in the case of an open channel. They showed that the $\gamma_{c_{\mathrm{u}}}$ value prescribed in Eurocode 7 is not sufficient to ensure the recommended minimum reliability index, i.e. its value should be increased. More specifically, for the $C O V_{c_{\mathrm{u}}}=0.3$, to reach $\beta \approx 3.8, \gamma_{c_{\mathrm{u}}} \approx 2.8$ is required. Similar results were presented by Forrest $\&$ Orr [3], on the example of a shallow foundation designed according to Eurocode 7.

This paper considers a shallow foundation which is centrally loaded with permanent action. In order to define the relevant random variables, the Sobol sensitivity analysis was conducted. The analysis determined the contribution of individual random variables to the total system response variance. The variables with a smaller Sobol index $(S I)$ value can be frozen. In this case, the overall factor of safety $(F S)$ was chosen for system response, and the analysis considered three random variables: undrained cohesion $\left(c_{\mathrm{u}}\right)$, permanent and $\left(V_{G}\right)$ i variable $\left(V_{Q}\right)$ load. The following Sobol indices values were obtained: $S I_{c_{\mathrm{u}}}=0.98, S I_{V_{\mathrm{G}}}=0.013$ and $S I_{V_{\mathrm{Q}}}=$ 0.007 . The results show that $c_{\mathrm{u}}$ is the dominant variable, whereas the contributions from $V_{G}$ and $V_{Q}$ are negligible. For the graphic presentation of the results, only two random variables were chosen $\left(c_{\mathrm{u}}\right.$ and $\left.V_{G}\right)$, while $V_{Q}$ was excluded from further analyses due to its negligible SI value.

The main goal of this paper is additional elaboration and calibration of the $\gamma_{c_{\mathrm{u}}}$ value prescribed in Eurocode 7, design approach 3. The partial factor is calibrated for the ULS (GEO) of shallow footing, RC2 reliability class and a 50-year reference period. To ensure a $\gamma_{c_{\mathrm{u}}}$ value that will provide the minimum recommended reliability index of 3.8 , calibration was performed for the critical combination of geometry, load, and soil parameters. Additional $\gamma_{c_{\mathrm{u}}}$ elaboration was proposed based on the uncertainty degree of $c_{\mathrm{u}}$ that is quantified by the coefficient of variation $\left(\mathrm{COV}_{c_{\mathrm{u}}}\right)$.

To meet the main goal, the following subsidiary goals are defined:

- the critical combination of geometry and load parameters determination using multiparameter analysis

- undrained shear strength variability quantification

- investigation of the influence of the marginal distribution of undrained shear strength on $\beta$ and $\gamma_{c_{u}}$

- selection of the appropriate calibration method
- comparison of results obtained using calibrated partial factor values, with the results obtained using partial factors prescribed in EC7, DA3.

\section{MATERIALS AND METHODS}

Calibration of $\gamma_{c_{\mathfrak{u}}}$ is performed in the case of the ultimate limit state (GEO) of a square footing under a permanent vertical load. The footing is analyzed in accordance with Eurocode 7, DA3. The influence of the force eccentricity on the $\beta$ value was also considered. In this case, a permanent vertical force, along with moments, is applied on the footing.

In all analyses, the following equality is satisfied: the design action is equal to the design resistance, i.e. $E_{\mathrm{d}}=R_{\mathrm{d}}$. The model geometry is shown in Fig. 1.

Reliability analyses are performed using the following assumptions:

- $\quad c_{\mathrm{u}}, \gamma$ and $E_{\mathrm{d}}$ are uncorrelated random variables

- foundation soil is coherent, homogeneous and isotropic

- the foundation soil is saturated.

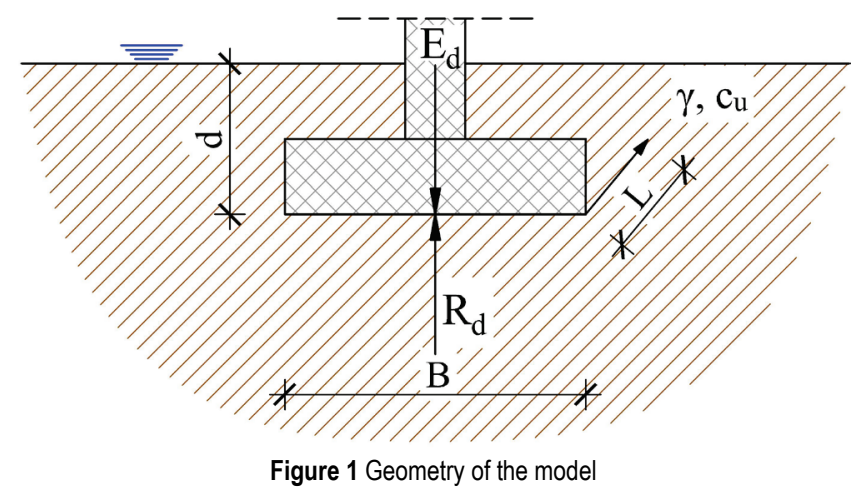

The First Order Reliability Method (FORM) was selected as the calibration method, and the design values of the random variables for partial factor calibration were determined from the graphical representation of the problem in the standard normal $(U)$ space. The above is practicable due to the number of random variables. The performance function is relatively simple, so it is expected that the iterative procedure within the FORM analysis will converge relatively quickly, with negligible error. Based on FORM results, the calibrated PF values were calculated as follows:

$\gamma_{c_{\mathrm{u}}}=\frac{c_{\mathrm{u}_{\mathrm{k}}}}{c_{\mathrm{u}_{\mathrm{d}}}}$

where: $\gamma_{c_{\mathrm{u}}}$ - undrained shear strength partial factor; $c_{\mathrm{u}_{\mathrm{d}}}$ the design value of $c_{\mathrm{u}} ; c_{\mathrm{u}_{\mathrm{k}}}$ - the characteristic value of $c_{\mathrm{u}}$.

Partial factor value for the permanent load is taken from Eurocode 7. 


\subsection{Random and Deterministic Variables in the Reliability Analysis}

The reliability integral of the problem consists of two random variables: undrained shear strength $\left(c_{\mathrm{u}}\right)$ and permanent vertical load $\left(V_{G}\right)$. The soil unit weight $(\gamma)$ is considered to be a deterministic variable since its prescribed partial factor $\left(\gamma_{\gamma}\right)$ value equals to one [15], i.e. $C O V_{\gamma} \approx 0$. Similarly, Murakami et al. [6] showed that $\gamma_{\gamma}$ for shallow foundation is in the range of 1-1.05. All other parameters are considered to be deterministic variables. The characteristics of random variables are shown in Tab. 3 .

Table 3 Characteristics of random variables
\begin{tabular}{|c|c|c|c|}
\hline $\begin{array}{c}\text { Random } \\
\text { variable }\end{array}$ & Designation & Statistical distribution & COV \\
\hline$V_{G}$ & $\mathrm{X} 1$ & Normal & 0.1 \\
\hline$c_{\mathrm{u}}$ & $\mathrm{X} 2$ & Normal, Lognormal* & changeable \\
\hline
\end{tabular}

* reliability analyses are performed both for normally and lognormally distributed $c_{\mathrm{u}}$

Due to low variability, a permanent load can be assumed to be normally distributed, and its characteristic value equals the mean [8].

Relevant literature gives different recommendations on the $c_{\mathrm{u}}$ statistical distribution. According to [16, 17], $c_{\mathrm{u}}$ is lognormally distributed, and the value of $C O V$ depends on the determination method. Hooper \& Butler [18] investigated the properties of London clays. Based on the histograms, they concluded that the probability density function (PDF) of $c_{\mathrm{u}}$ is probably normal. Murakami et al. [6] concluded the same, but in the case of large variability, they recommend the use of lognormal PDF. Reliability analyses with both (normal and lognormal) assumptions were performed in this paper.

Characteristic values of geotechnical parameters were calculated from the mean values using Eq. 2 [19]. Corresponding design values are determined according to EC7, DA3 [15].

$x_{\mathrm{k}}=v_{\mathrm{m}} \cdot\left(1-\frac{C O V_{x}}{2}\right)$

where: $x_{\mathrm{m}}$ - is the mean value of $X ; C O V_{x}$ - is the coefficient of variation of $X$.

\subsection{Reliability Integral}

The reliability integral is approximated using First Order Reliability Analysis (FORM). Because of a relatively simple mathematical expression of the performance function, relatively fast convergence and small error are expected.

\subsubsection{Performance Function (PF)}

Performance function is defined as follows [20]:

$g=R-S$ where $R=r(R)$, and $S=s(s)$ - random variables related to resistances and actions.

In the case of a shallow foundation, $R$ and $S$ can be expressed in the following way [15]:

$$
\begin{aligned}
& S=E_{\mathrm{d}}=\gamma_{G} \cdot V_{G}=1.35 \cdot V_{G} \\
& R=\frac{R_{\mathrm{k}}}{\gamma R_{\mathrm{v}}}=R_{\mathrm{d}}=\left[(\pi+2) \cdot c_{\mathrm{u}, \mathrm{d}} \cdot s_{\mathrm{c}} \cdot i_{\mathrm{c}}+q\right] \cdot A^{\prime}
\end{aligned}
$$

where: $E_{\mathrm{d}}$ - design value of the effect of actions, $V_{G}$ characteristic value of permanent load, $\gamma_{G}$ - partial factor for a permanent action, $c_{\mathrm{u}, \mathrm{d}}$ - undrained shear strength design value; $s_{\mathrm{c}}, i_{\mathrm{c}}$ - dimensionless factors for the shape of the foundation and the inclination of the load; $q$ - the design total overburden pressure at the level of the foundation base.

Dimensionless factors $s_{\mathrm{c}}$ and $i_{\mathrm{c}}$ are calculated using equations from [15].

Substituting the terms in the expressions (3) with the expressions (4) and (5), and by replacing the designation of random variables in accordance with Tab. 3 , the performance function in the physical space ( $X$ space) yields:

$$
g\left(x_{1}, x_{2}\right)=\left[(\pi+2) \cdot x_{2} \cdot s_{\mathrm{c}} \cdot i_{\mathrm{c}}+q\right] \cdot A^{\prime}-x_{1}
$$

To simplify the reliability analysis, random variables are transformed from the $X$ space to the standard normal space ( $U$ space). The Nataf transformation is used to construct a normal cumulative probability density function (Nataf model) by transforming original variables into standard normal variables [21]. The Nataf transformation is an approximate method, for which the following input parameters are required: the covariance matrix and marginal cumulative density functions (CDF) of random variables [22, 23]. Because all variables are uncorrelated, and either normal or lognormal, the Nataf transformation is very efficient. Since analyses are performed with the assumptions that $c_{u}$ is both normally and lognormally distributed, results of the transformations are two performance functions in the standard normal space, Eq. (7) and Eq. (8).

$$
\begin{aligned}
& g\left(x_{1}, x_{2}\right) *=\left[(\pi+2) \cdot\left(\mu_{x_{2}}+\sigma_{x_{2}} \cdot u_{2}\right) \cdot b_{\mathrm{c}} \cdot s_{\mathrm{c}} \cdot i_{\mathrm{c}}+q\right] \cdot A^{\prime}- \\
& -\left(\mu_{x_{1}}+\sigma_{x_{1}} \cdot u_{1}\right) \\
& g\left(x_{1}, x_{2}\right)=\left[(\pi+2) \cdot \mathrm{e}^{\lambda_{2}+\xi_{2} \cdot u_{2}} \cdot b_{\mathrm{c}} \cdot s_{\mathrm{c}} \cdot i_{\mathrm{c}}+q\right] \cdot A^{\prime}- \\
& -\left(\mu_{x_{1}}+\sigma_{x_{1}} \cdot u_{1}\right)
\end{aligned}
$$

where: $u_{i}=\frac{x_{i}-\mu_{x_{i}}}{\sigma_{x_{i}}}$ - transformed random variable; $\mu_{x_{i}}$ mean value of the random variable $x_{i} ; \sigma_{x_{i}}$ - standard deviation of the random variable $x_{i} ; \lambda_{2}, \xi_{2}$ - lognormal distribution parameters of variable $x_{2}$. 


\subsubsection{Integrand}

Since two different analyses are performed, it is necessary to define two reliability integrals, and thus two integrands. Both integrands were transformed into the standard normal space using Nataf transformations. In the standard normal space, their contours are concentric circles. The first integrand is a bivariate joint PDF (Eq. 9), and the second hybrid joint PDF of the normal and lognormal distribution (Eq. 11).

$$
\begin{aligned}
& \tilde{f}_{x_{1} x_{2}}\left(x_{1}, x_{2}\right) *= \\
& *=\frac{1}{2 \pi \sqrt{1-\rho_{12}^{2}}} \exp \left\{-\frac{1}{2\left(1-\rho^{2}\right)}\left[u_{1}^{2}-2 \rho_{12} u_{1} u_{2}-u_{2}^{2}\right]\right\}
\end{aligned}
$$

where:

$$
u_{i}=\frac{x_{i}-\mu_{x_{i}}}{\sigma_{x_{i}}}
$$

$\rho_{12}$ - correlation coefficient between $x_{1}$ and $x_{2}$

$$
\begin{aligned}
& \tilde{f}_{x_{1} x_{2}}\left(x_{1}, x_{2}\right) *= \\
& *=\frac{1}{2 \pi \sigma_{1} \xi_{1} \sqrt{1-\rho_{12}^{2} x_{2}}} \exp \left\{-\frac{1}{2}\left[\frac{u_{2}-\eta_{2} u_{1}}{\sqrt{1-\eta_{2}^{2}}}\right]^{2}--\frac{1}{2} u_{1}^{2}\right\}
\end{aligned}
$$

where:

$$
\begin{aligned}
& u_{1}=\frac{x_{1}-\mu_{x_{1}}}{\sigma_{x_{1}}} \\
& u_{2}=\frac{\ln \left(x_{2}\right)-\lambda_{\mathrm{s}}}{\sigma_{x_{1}}} \\
& \eta_{2}=\frac{\rho_{12} \kappa_{2}}{\xi_{2}}
\end{aligned}
$$

$\rho_{12}$ - correlation coefficient between $x_{1}$ and $x_{2}$

Reliability integrals for the normally and lognormally distributed $c_{\mathrm{u}}$ are shown in Eq. 15 and Eq. 16, respectively.

$$
\begin{aligned}
& P_{f}=\int_{g\left(x_{1}, x_{2}\right) *<0} \tilde{f}_{x_{1} x_{2}}\left(x_{1}, x_{2}\right) * \mathrm{~d} x_{1} \mathrm{~d} x_{2} \\
& P_{f}=\int_{g\left(x_{1}, x_{2}\right)<0} \tilde{f}_{x_{1} x_{2}}\left(x_{1}, x_{2}\right) \mathrm{d} x_{1} \mathrm{~d} x_{2}
\end{aligned}
$$

\subsection{First Order Reliability Method (FORM)}

The method was developed by [24], to address the main shortcomings of the First Order Second Moment (FOSM) method. Unlike the FOSM, in which limit state function is linearized at the point where all variables have a mean value, in the FORM, it is linearized at the point on the failure surface (or linearized at point $\mathrm{A}$, or at the point $\mathrm{OF}$ ). The procedure includes the random variables and limit state function transformation from the physical to the standard normal space. Rosenblatt or Nataf transformations can be used for this purpose [25]. Reliability analysis using FORM requires knowledge of statistical distributions of random variables.

In this paper, the Nataf transformation is used for transformations of random variables and the limit state function into the standard normal space. Then, the reliability index $(\beta)$ is calculated performing the iterative procedure shown in Fig. 2. The reliability index is defined as the shortest distance from the origin to the failure surface (Fig. 3 ). The point on the failure surface closest to the origin is named the Most probable Point (MPP). FORM analyses were performed using a script written in the MATLAB programming language.

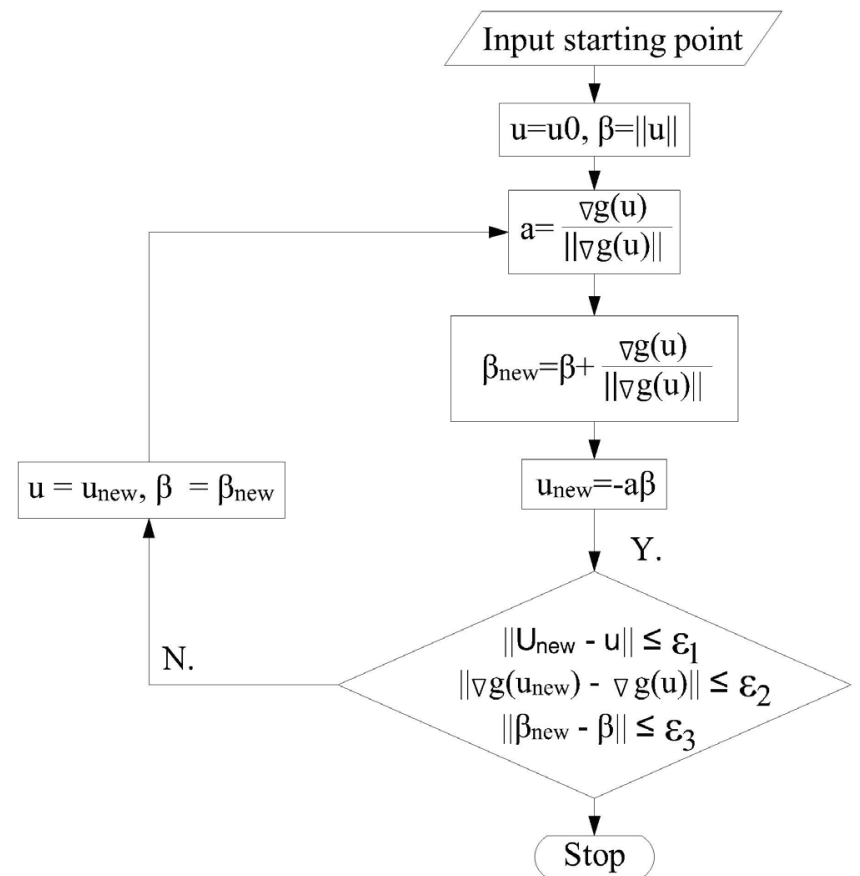

Figure 2 Flowchart of the FORM [26]

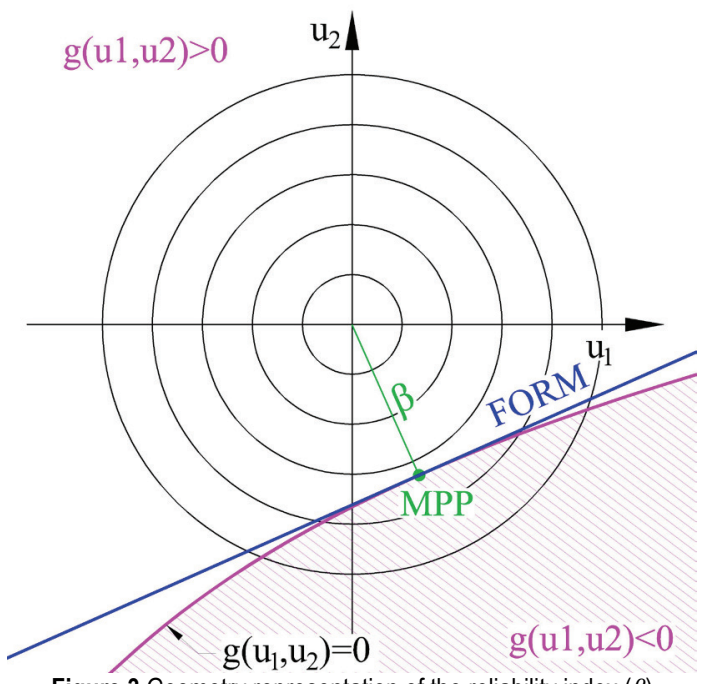

Figure 3 Geometry representation of the reliability index $(\beta)$ 


\subsection{Geotechnical Parameter Degree of Variability Quantification}

Degree of geotechnical parameter variation is quantified by the values of the corresponding coefficient of variation $(\mathrm{COV})$. In this paper, the classification by Phoon [14] is adopted (Tab. 2). Values of $C O V$ are divided into 3 classes, and reliability indexes were calculated for the mean of each class. Regarding the undrained shear strength, low, medium, and high variability corresponds to the following $\mathrm{COV}_{c_{\mathrm{u}}}$ ranges: $10-30 \%, 30-50 \%$ and $50-70 \%$ respectively.

\subsection{Partial Factor Calibration}

The undrained shear strength partial factor $\left(\gamma_{c_{u}}\right)$ is calibrated using the First Order Reliability Method (FORM). Calibration was performed iteratively, by conducting a series of reliability analyses to determine $\gamma_{c_{\mathrm{u}}}$, which corresponds to the recommended minimum reliability index value of 3.8 . Analyses were performed using a script written in the MATLAB programming language.

\section{RESULTS AND DISCUSSION}

\subsection{Results of Multiparameter Analyses}

Multiparameter analyses results are shown in Figs. 4 and 5. Influences of foundation geometry, load eccentricity, and $C O V_{c_{\mathrm{u}}}$ on the reliability index value were examined. Analyses were performed for $C O V_{c_{\mathrm{u}}}=0.2,0.4$, and 0.6, but only the results for the case of $\mathrm{COV}_{c_{\mathrm{u}}}=0.2$ are presented. The reason are similar trends obtained from the other two analyses. The influence of load eccentricity on the $\beta$ value is shown in Fig. 4. The difference between the extreme $\beta$ values is in the $3^{\text {rd }}$ decimal place, so its influence was excluded from further consideration. The $B / L$ ratio also has no significant impact on $\beta$ (Fig. 5). The depth of the foundation base has a significant effect on the $\beta$ value, which can be seen in Fig. 5. Regarding the foundation reliability index, the most unfavorable depth is $d=0$. The reason for this is the absence of the total overburden pressure from soil bearing capacity, at the foundation base level.

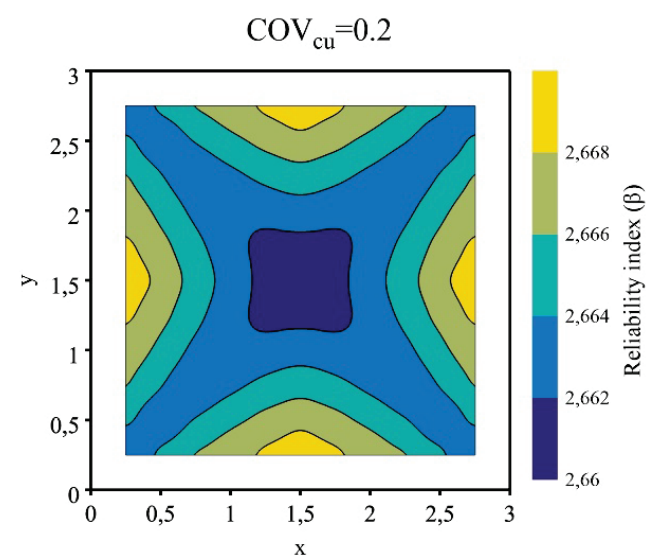

Figure 4 The influence of the load eccentricity on $\beta$ value

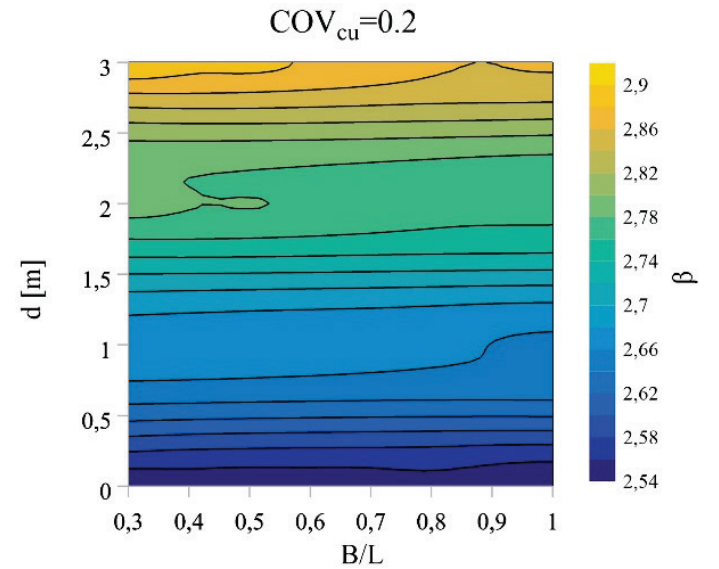

Figure 5 The influence of $B / L$ ratio and the foundation base depth on $\beta$ value

Based on the multiparameter analyses results, the foundation geometry and the load position used in the partial factor calibration are selected as follows: $B=L=3.0 \mathrm{~m}, d=$ $0 \mathrm{~m}$ and $e_{x}=e_{y}=0 \mathrm{~m}$.

\subsection{The Influence of Marginal Statistical Distribution of $c_{\mathrm{u}}$ Strength on $\beta$ Value}

Results of the reliability analyses with different statistical distributions of the $c_{\mathrm{u}}$ are shown in Figs. 6, 7, and 8 . The limit state function $g_{*}(U)=0$ (magenta) represents the case of normally distributed $c_{\mathrm{u}}$, and the $g(U)=0$ (green) the case of lognormally distributed $c_{\mathrm{u}}$. The trend of decreasing $\beta$ by increasing $C O V_{c_{\mathrm{u}}}$ is visible when comparing their values from Figs. 6, 7 and 8, which is within expectations. Also noticeable is a significant difference between the $\beta$ values obtained with normally and lognormally distributed $c_{\mathrm{u}}$.

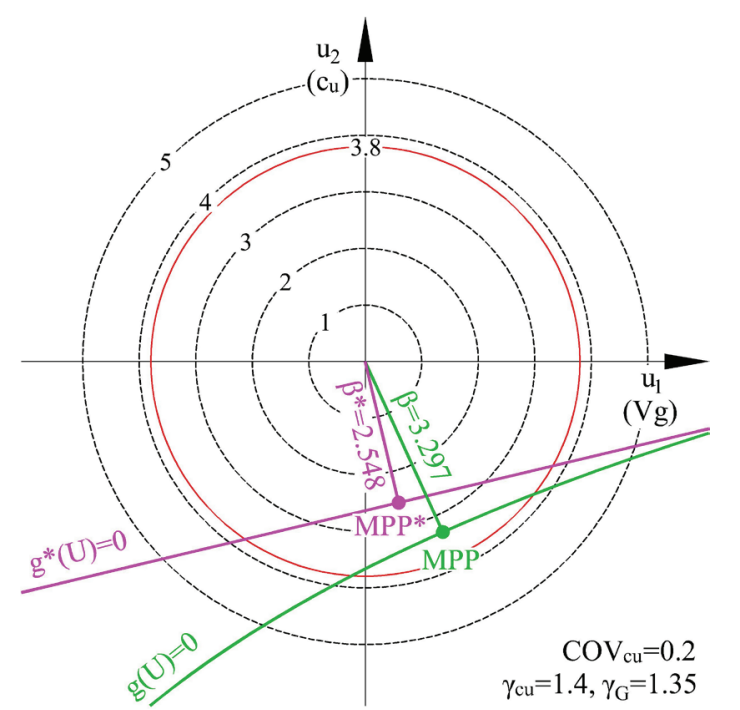

Figure 6 Comparison between reliability indexes, calculated with normally and lognormally distributed $c_{\mathrm{u}}$, case $\operatorname{COV}_{c_{\mathrm{u}}}=0.2$

The differences between reliability indexes calculated using normally and lognormally distributed $c_{\mathrm{u}}$ are shown in Tab. 4. 
Table 4 The difference between reliability indexes, calculated using normally and lognormally distributed $c_{\mathrm{u}}$

\begin{tabular}{|c|c|c|}
\hline$C O V_{c_{\mathrm{u}}}$ & $|\Delta \beta|($ absolute value $)$ & $\beta_{\text {lognormal }} / \beta_{\text {normal }}$ \\
\hline 0.2 & 0.749 & 1.29 \\
\hline 0.4 & 0.542 & 1.38 \\
\hline 0.6 & 0.444 & 1.42 \\
\hline
\end{tabular}

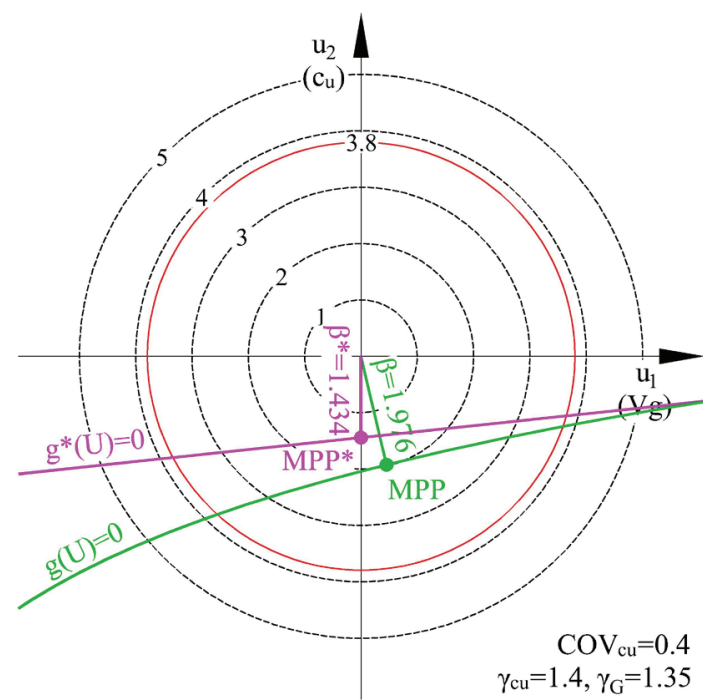

Figure 7 Comparison between reliability indexes, calculated with normally and lognormally distributed $c_{\mathrm{u}}$, case $C O V_{c_{\mathrm{u}}}=0.4$

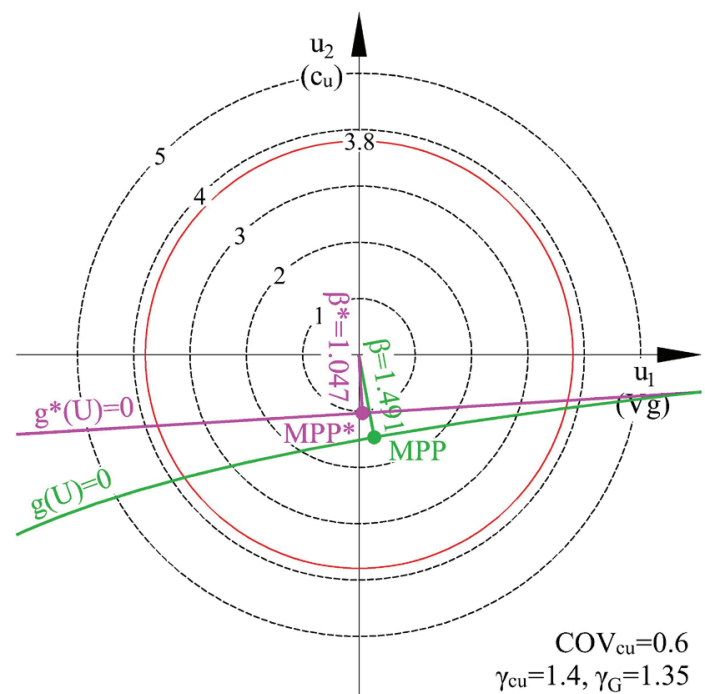

Figure 8 Comparison between reliability indexes, calculated with normally and lognormally distributed $c_{\mathrm{u}}$, case $C O V_{\mathrm{c}_{\mathrm{u}}}=0.6$

\subsection{Partial Factor Calibration $\left(\gamma_{c_{\mathrm{u}}}\right)$}

Undrained shear strength partial factor was calibrated for a normally and lognormally distributed $c_{\mathrm{u}}$. Calibration results are shown in Fig. 9.

The results obtained using normally distributed $c_{\mathrm{u}}$ are not presented, because even in the case of low $\operatorname{COV}_{c_{\mathrm{u}}}, \gamma_{c_{\mathrm{u}}}$ values are unreasonably high, and therefore not applicable in practice. The same conclusion was presented by Murakami et al. [6]. A trend of increasing $\gamma_{c_{\mathrm{u}}}$ with the $C O V_{c_{\mathrm{u}}}$ increase is visible in Fig. 9. All presented values are higher than those prescribed in EC7, and their application would result in a significantly more conservative design, compared to current practice.

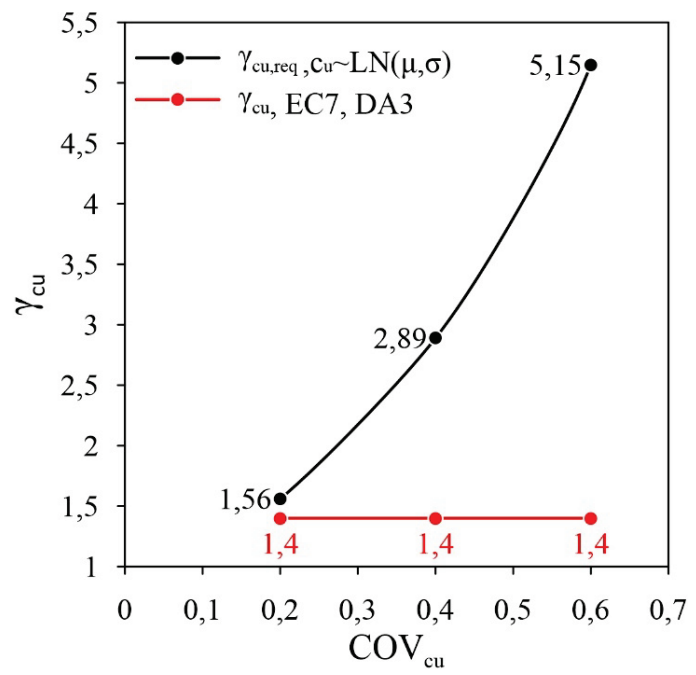

Figure 9 The results of $\gamma_{c_{u}}$ calibration

\section{NUMERICAL EXAMPLE}

Analyses of shallow footings using undrained shear strength partial factors, presented in Fig. 9, were performed. The main goal was to determine the required footing width, such that the ultimate limit state (GEO) is satisfied. The results were compared to the footing width obtained by the analysis performed with $\gamma_{c_{\mathrm{u}}}=1.4$ [15]. The partial factor applied to the permanent load in all cases is equal to 1.35 . Geometry of the problem, along with the load and geotechnical parameters, are shown in the Fig. 10. Three cases regarding the $C O V_{c_{\mathrm{u}}}$ were analyzed: $C O V_{c_{\mathrm{u}}}=0.2,0.4$ and 0.6 .

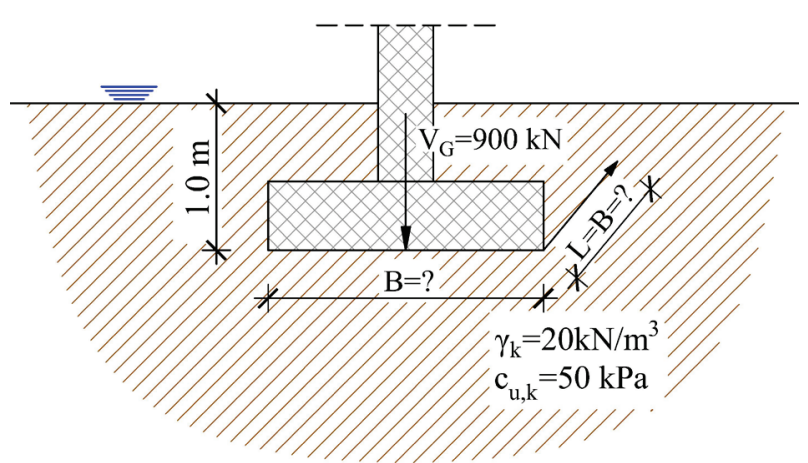

Figure 10 Geometry of the problem

Tab. 4 shows results of the four ultimate limit state analyses, which were performed in accordance with Eurocode 7, design approach 3. A deviation from the calculation procedure was made in analyses 2-4. Instead of the prescribed $\gamma_{c_{u}}$ value, values shown in Fig. 9 were used. As expected, a positive trend between $\mathrm{COV}_{c_{\mathrm{u}}}$ and the required footing width can be seen. 
As it can be seen from Tab. 5, the footing width is the function of the $c_{\mathrm{u}, \mathrm{d}}$, which is calculated using the corresponding partial factor, $\gamma_{c_{\mathrm{u}}}$. The purpose of $\gamma_{c_{\mathrm{u}}}$ is to consider the soil's natural variability, along with uncertainties of various origin. In the ultimate limit state analysis, the aforementioned can be quantified by the coefficient of variation, $\mathrm{COV}$. This way, its value can provide a meaningful tool for additional elaboration of partial factors.

Table 5 Required footing widths for different $\gamma_{c_{u}}$ values

\begin{tabular}{|c|c|c|c|c|}
\hline No. & Analysis & $\gamma_{c_{\mathrm{u}}}$ & $c_{\mathrm{u}, \mathrm{d}}{ }^{*}$ & $B_{\text {required }}$ \\
\hline 1 & EC7, DA3 & 1.4 & 35.71 & 2.25 \\
\hline 2 & $\operatorname{COV}_{c_{u}}=0.2$ & 1.56 & 28.85 & 2.48 \\
\hline 3 & $\operatorname{COV}_{c_{u}}=0.4$ & 2.89 & 13.84 & 3.40 \\
\hline 4 & $\operatorname{COV}_{c_{u}}=0.6$ & 5.15 & 6.80 & 4.43 \\
\hline
\end{tabular}

* design value of undrained shear strength

\section{DISCUSSION}

With the goal of additional elaboration and calibration of the $\gamma_{c_{\mathrm{u}}}$ value prescribed in Eurocode 7, reliability analyses were performed. Calibration was performed in the case of a footing ULS (GEO) analysis under a permanent vertical load. To meet the minimum recommended $\beta$ value, analyses were carried out for the critical combination of geometry, load, and statistical distribution of $c_{\mathrm{u}}$. Reliability class $\mathrm{RC} 2$ and a reference period of 50 years were considered. Corresponding recommended minimum value of reliability index equals to 3.8 [8]. Analogous to Canadian Standards Organization (2014), $\gamma_{c_{\mathrm{u}}}$ is divided into three ranges concerning the $C O V_{c_{\mathrm{u}}}$ value. Because of a relatively simple form of the performance function, the First Order Reliability Method (FORM) was selected as a method for reliability index calculation.

Reliability analysis with normally distributed $c_{\mathrm{u}}$ resulted in unreasonably low $\beta$; therefore, these results were not considered in partial factor calibration. They were calibrated only using a $\operatorname{lognormally}$ distributed $c_{\mathrm{u}}$. A similar conclusion was presented by Murakami et al. [6] in reliability analyses of a shallow foundation under open channels.

It is shown that even in the case of $\mathrm{COV}_{c_{\mathrm{u}}}=0.2$ (Fig. 6), the reliability index of a shallow footing is lower than the recommended minimum value. To achieve this value, $C O V_{c_{\mathrm{u}}}=0.17$ is required. It is therefore concluded that $C O V_{c_{\mathrm{u}}} \leq 0.17$ will ensure that footings designed in accordance with EC7, DA3 will meet the minimum target reliability index. According to $[6,27,28] \mathrm{COV}_{c_{\mathrm{u}}}$ ranges from 15 to $40 \%$, depending on the natural variability of the soil, the method of measurement, and the transformation model. Such a wide range of possible values, i.e. high uncertainty, is supporting the idea of additional elaboration of partial factors to achieve a reasonably constant reliability level.

Instead of a single value of the partial factor $\gamma_{c_{\mathrm{u}}}$, three values with respect to $C O V$ are proposed (Tab. 6).
Table 6 Required partial factors of undrained shear strength for different $C O V_{c_{u}}$ values

\begin{tabular}{|c|c|}
\hline \multicolumn{2}{|c|}{ values } \\
\hline$C O V_{c_{\mathrm{u}}}$ & $\gamma_{c_{\mathrm{u}}}$ \\
\hline 0.2 & 1.56 \\
\hline 0.4 & 2.89 \\
\hline 0.6 & 5.15 \\
\hline
\end{tabular}

The proposed values are valid only in the case of Design Approach 3. An alternative to the additional elaboration of partial factors may be to reduce the minimum recommended $\beta$ value in the range between 3-3.5.

That way, footings designed using the currently prescribed $\gamma_{c_{\mathrm{u}}}$ value would satisfy the criteria regarding the minimum recommended reliability index. According to [11] reliability index in a range of 3.1-3.7 would provide a satisfactory reliability level, and according to [29], $\beta>3$ ensures above-average structure performance level.

\section{CONCLUSION}

The absence of consideration of soil variability in geotechnical analyses can lead to unreliable results, and consequently to constructions with reliability values lower than required. The unique value of the partial factor of the geotechnical parameter does not reflect the actual state of site-specific variabilities in a specific task. Using reliability analyses, we have demonstrated that higher variability requires a greater partial factor in order to ensure meeting the required reliability level. Introducing an additional classification of partial factors of geotechnical parameters (with the respect to the coefficient of variation) would enable designers to adjust their designs to actual soil conditions. This would ensure a similar level of design reliability for all geotechnical constructions. For further research, we suggest a calibration, and an additional classification of the partial factors of the remaining geotechnical parameters, for different types of geotechnical tasks.

\section{REFERENCES}

[1] JCSS. (2006). Joint Committee on Structural Safety, JCSS Probabilistic Model Code, Section 3.7: Soil Properties.

[2] Abd Alghaffar, M. A. \& Dymiotis-Wellington, C. (2005). Reliability analysis of retaining walls designed to British and European standards. Structure and Infrastructure Engineering, 1(4), 271-284. https://doi.org/10.1080/15732470500030455

[3] Forrest, W. S. \& Orr, T. L. L. (2010). Reliability of shallow foundations designed to Eurocode 7. Georisk, 4(4), 186-207. https://doi.org/10.1080/17499511003646484

[4] Foye, K. C., Salgado, R., \& Scott, B. (2006). Resistance factors for use in shallow foundation LRFD. Journal of Geotechnical and Geoenvironmental Engineering, 132(9), 1208-1218. https://doi.org/10.1061/(ASCE)1090-0241(2006)132:9(1208)

[5] Lesny, K. (2019). Probability-based derivation of resistance factors for bearing capacity prediction of shallow foundations under combined loading. Georisk, 13(4), 284-290. https://doi.org/10.1080/17499518.2019.1633581

[6] Murakami, A., Nishimura, S. I., Suzuki, M., Mori, M., Kurata, T., \& Fujimura, T. (2011). Determination of partial factors for the verification of the bearing capacity of shallow foundations under open channels. Georisk, 5(3-4), 186-194. 
https://doi.org/10.1080/17499518.2011.558417

[7] Meinen, N. E. \& Steenbergen, R. D. J. M. (2018). Reliability levels obtained by Eurocode partial factor design - A discussion on current and future reliability levels. Heron, 63(3), 243-302.

[8] EN. (2011). European Committee for Standardization, Eurocode: Basis of structural design (EN 1990:2002+A1:2005+A1:2005/AC:2010).

[9] Simpson, B. (2017). Eurocode 7 and Robustness.Geotechnical Special Publication, 7(GSP 282), 52-68. https://doi.org/10.1061/9780784480694.004

[10] Abd Alghaffar, M. A. \& Dymiotis-Wellington, C. (2007). Time-variant reliability of retaining walls and calibration of partial factors. Structure and Infrastructure Engineering, 3(3), 187-198. https://doi.org/10.1080/15732470500440845

[11] Meyerhof, G. G. (1970). Safety factors in soil mechanics. Canadian Geotechnical Journal, 7(4), 349-355. https://doi.org/10.1139/t70-047

[12] CWC. (2014). Canadian Standards Organization, Canadian highway bridge design code.

[13] Paikowsky, S. G., et al. (2004). NCHRP REPORT 507, vol. 10. Washington, D.C.: Transportation Research Board.

[14] Phoon, K.-K. (2008). Reliability-based design in geotecnical engineering, $1^{\text {st }}$ ed. New York: Taylor \& Francis Geological. https://doi.org/10.1201/9781482265811

[15] EN. (2012). European Committee for Standardization, Eurocode 7: Geotechnical design -- Part 1: General rules (EN 1997-1:2004+AC:2009).

[16] Lacasse, S. \& Nadim, F. (2007). Probabilistic geotechnical analyses for offshore facilities. Georisk, 1(1), 21-42. https://doi.org/10.1080/17499510701204224

[17] Wang, Y., Akeju, O. V., \& Cao, Z. (2016). Bayesian Equivalent Sample Toolkit (BEST): an Excel VBA program for probabilistic characterisation of geotechnical properties from limited observation dana. Georisk, 10(4), 251-268. https://doi.org/10.1080/17499518.2016.1180399

[18] Hooper, J. A. \& Butler, F. G. (1966). Some numerical results concerning the shear strength of london clay. Geotechnique, 16(4), 282-304. https://doi.org/10.1680/geot.1966.16.4.282

[19] Schneider, H. R. (1999). Definition and determination of characteristic soil properties. Fourteenth International Conference on Soil Mechanics and Foundation Engineering. Proceedings, 2271-2274.

[20] JCSS. (2001). Joint Committee on Structural Safety, Probabilistic model code Part 1 - Basis of Design.

[21] Choi, K. K., Noh, Y., \& Du, L. (2007). Reliability based design optimization with correlated input variables. SAE Technical Papers. https://doi.org/10.4271/2007-01-0551

[22] Ditlevsen, O. \& Madsen, H. O. (1996). Structural Reliability Methods. Chichester: John Wiley \& Sons.

[23] Melchers, R. E. \& Bect, A. T. (2018). Structural reliability analysis and prediction, $3^{\text {rd }}$ ed. New York: Wiley. https://doi.org/10.1002/9781119266105

[24] Hasofer, A. M. \& Lind, N. C. (1974). An exact and invariant first order reliability format. Journal Eng. Mech. Division (ASCE), 100, 111-121. https://doi.org/10.1061/JMCEA3.0001848

[25] Lebrun, R. \& Dutfoy, A. (2009). Do Rosenblatt and Nataf isoprobabilistic transformations really differ? Probabilistic Engineering Mechanics, 24(4), 577-584. https://doi.org/10.1016/j.probengmech.2009.04.006

[26] Xiaoping, D. (2005). Probabilistic Engineering Design, First Order and Second Reliability Methods (Lecture notes). University of Missouri - Rolla.

[27] Duncan, J. M. (2000). Factors of Safety and Reliability in Geotechnical Engineering. Journal of Geotechnical and
Geoenvironmental Engineering, 126, 307-316.

https://doi.org/10.1061/(ASCE)1090-0241(2000)126:4(307)

[28] Phoon, K.-K. \& Kulhawy, F. H. (1999). Characterization of geotechnical variability. Canadian Geotechnical Journal, 36(4), 612-624. https://doi.org/10.1139/t99-038

[29] USACE. (1997). U.S. Army Corps of Engineers, Engineering and Design - Introduction to probability and reliability methods for use in geotechnical engineering, no. 1110-2-547. Washington DC.

\section{Authors' contacts:}

\section{Filip Dodigović}

(Corresponding author)

Faculty of Geotechnical Engineering, University of Zagreb,

Hallerova aleja 7, 42000 Varazdin, Croatia

filip.dodigovic@gmail.com

\section{Krešo Ivandić}

Faculty of Geotechnical Engineering, University of Zagreb,

Hallerova aleja 7, 42000 Varazdin, Croatia

kivandic@gfv.hr

\section{Božo Soldo}

University North, Department of Civil Engineering,

Jurja Križanića 31b, 42000 Varaždin, Croatia

bsoldo@unin.hr

Kristijan Grabar

SPP d.o.o.

Davora Trstenjaka 3, 42000 Varaždin, Croatia

kristijan@grabar.biz 